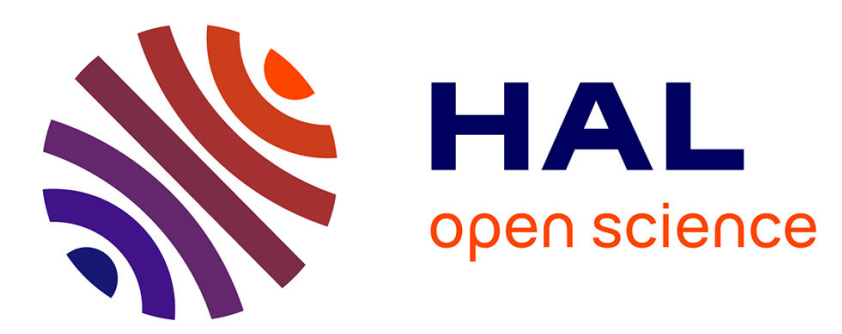

\title{
Stationary responses of gear systems with combined periodically time-varying stiffness and stochastic external excitations
}

\author{
Joël Perret Liaudet, Pierre Garambois, Emmanuel Rigaud
}

\section{To cite this version:}

Joël Perret Liaudet, Pierre Garambois, Emmanuel Rigaud. Stationary responses of gear systems with combined periodically time-varying stiffness and stochastic external excitations. MPT2017 - Motion Power Transmission, Feb 2017, Kyoto, Japan. hal-01484815

\section{HAL Id: hal-01484815 https://hal.science/hal-01484815}

Submitted on 7 Mar 2017

HAL is a multi-disciplinary open access archive for the deposit and dissemination of scientific research documents, whether they are published or not. The documents may come from teaching and research institutions in France or abroad, or from public or private research centers.
L'archive ouverte pluridisciplinaire HAL, est destinée au dépôt et à la diffusion de documents scientifiques de niveau recherche, publiés ou non, émanant des établissements d'enseignement et de recherche français ou étrangers, des laboratoires publics ou privés. 


\title{
STATIONARY RESPONSES OF GEAR SYSTEMS \\ WITH COMBINED PERIODICALLY TIME-VARYING STIFFNESS AND STOCHASTIC EXTERNAL EXCITATIONS
}

\author{
* Joël PERRET-LIAUDET Lab. Tribologie et Dynamique des Systèmes, École centrale de Lyon, UMR 5513 \\ Université de Lyon, Écully cedex F69134, FRANCE \\ E-mail: joel.perret-liaudet@ec-lyon.fr \\ Pierre GARAMBOIS Lab. Tribologie et Dynamique des Systèmes, École centrale de Lyon, UMR 5513 \\ Université de Lyon, Écully cedex F69134, FRANCE \\ E-mail: pierre.garambois@ec-lyon.fr \\ Emmanuel RIGAUD Lab. Tribologie et Dynamique des Systèmes, École centrale de Lyon, UMR 5513 \\ Université de Lyon, Écully cedex F69134, FRANCE \\ E-mail: emmanuel.rigaud@ec-lyon.fr
}

Keywords: Geared transmission, parametric excitation, periodic stiffness, stochastic excitation.

\begin{abstract}
The stationary dynamic response of a geared system with time-varying mesh stiffness subjected to stationary stochastic external excitation is examined in this paper. To this end, a spur gear pair model with periodical timevarying mesh stiffness is extended to include stochastic external force excitations. These random forces are introduced as second-order stationary and ergodic processes. In order to compute the stationary response, a very efficient method, called the iterative spectral method is used. This method is derived in the frequency domain and provides the explicit power spectral density of the response. The PSD response is expressed as a function of the bispectrum which is the bilinear Fourier transform of the bitemporal impulse response of the parametric system.
\end{abstract}

\section{INTRODUCTION}

The static transmission error is the main internal source of gear dynamics, in particular in the case of the noise radiated by these systems [1-2]. Static transmission error can be viewed as the difference between the actual position of the driven gear and its theoretical position for a very slow rotation speed and for a given applied torque. Its characteristics depend on the instantaneous contacts of the meshing tooth pairs which result from tooth deflections and actual tooth surface micro-level geometry. In order to model this internal source, the elastic forces between gears are usually introduced by a combination of time-periodic stiffnesses and an imposed displacement function, both acting along the lines of action. The steady state dynamic responses of this kind of model with a constant external load are extensively studied [3], with in mind the problem of the whining noise [4].

Geared systems are sometimes excited by external excitations; see for example the cases of rattle noise and hammering noise induced by the acyclism of the engine [5$6]$. With this type of problem, few studies have focused on the case of the dynamic response under stationary stochastic external excitations. However, a number of practical cases can be considered such as geared systems subjected to frictional forces, wind flow for windmill, fluid load for vacuum pumps, to cite a few.

In this context, the stationary dynamic response of a geared system with time-varying mesh stiffness subjected to stationary stochastic external excitation is examined in this work. For this purpose, a parallel gear pair model with a periodic time-varying mesh stiffness is extended to include stochastic external force excitations. The latest are introduced as a second-order stationary and ergodic random process. This paper starts with the description of this modelling.

\section{DYNAMIC MODELLING}

\section{Equations of motion}

A standard single stage parallel gear pair running in stationary regimes is considered here. In this study, secondorder stationary and ergodic random processes are assumed to be part of the external excitations. Considering a multidegree-of-freedom periodic-time-varying linearized system, the governing equations of motion can be written in the matrix form as

$$
\mathbf{M} \ddot{\mathbf{x}}+\mathbf{C} \dot{\mathbf{x}}+\mathbf{K}(t) \mathbf{x}=\mathbf{f}(t)+\mathbf{w}(t)
$$

In this equation, $\mathbf{x}(t)$ is the generalized displacement vector. $\mathbf{M}$ is the time-invariant mass matrix. $\mathbf{C}$ is the timeinvariant damping matrix. It is assumed to be a proportional or Rayleigh damping matrix with respect of the timeaveraged mass and stiffness matrices. $\mathbf{K}(t)$ is a periodically time-varying matrix. The time variation is induced by the time varying mesh stiffness acting between pinions. Usually, this variation is $T_{z}$-periodic at the gear mesh frequency $f_{z}=1 / T_{z}$. The external excitation terms consist on a classic force vector $\mathbf{f}(t)$ and a random process $\mathbf{w}(t)$ introduced for the present purpose. The first one which includes the internal static transmission error excitations will be excluded in this study according to the principle of linearity. The second one may include internal and external random excitations. This process is introduced through the knowledge of its power spectral density:

$$
\mathbf{S}_{\mathbf{w w}}(\omega)=\lim _{T \rightarrow \infty} \mathbf{S}_{\mathbf{w w}}(\omega, T)
$$

where

$$
\mathbf{S}_{\mathbf{w w}}(\omega, T)=\lim _{N \rightarrow \infty} \frac{1}{N} \sum_{k=1}^{N} \mathbf{S}_{\mathbf{w w}}^{(k)}(\omega, T)
$$


is the power spectral density estimator of the truncated process defined over finite duration $T$. Consider sample functions or time histories $\mathbf{w}_{T}^{(k)}(t)$ of the truncated process $\left\{\mathbf{w}_{T}(t)\right\}$ from the original ones, and $\mathbf{W}_{T}^{(k)}(\omega)$ its Fourier transform with respect to $t$, we have

$$
\mathbf{S}_{\mathbf{w w}}^{(k)}(\omega, T)=\mathbf{W}_{T}^{(k)}(\omega) \mathbf{W}_{T}^{(k) *}(\omega)
$$

where $\mathbf{A}^{*}$ denotes the transpose conjugate of $\mathbf{A}$.

\section{Power spectral density of responses}

Preliminary, it is assumed that the studied system is asymptotically stable in the Lyapunov sense, i.e. damping is sufficient to prevent parametric instabilities. In this case, the free response decreases asymptotically toward $\mathbf{0}$, the equilibrium state.

Consider now forced response $h_{i j}(\tau, t)$ caused at the $i$-th degree-of-freedom at time $t$ by an unit impulse force $\delta_{j}(t-\tau)$ at the $j$-th degree-of-freedom of the system at time $(t-\tau)$. These impulse responses constitute the bitemporal matrix $\mathbf{h}(\tau, t)$ which relates the output response $\mathbf{x}(t)$ to the input process, for example $\mathbf{y}(t)$, as:

$$
\mathbf{x}(t)=\int \mathbf{h}(\tau, t) \mathbf{y}(t-\tau) \mathrm{d} \tau
$$

By assuming $h_{i j}(\tau, t)$ functions to be physically realizable, the bilinear Fourier transform with respect to times $\tau$ and $t$ is written in the matrix form as follows:

$$
\mathbf{H}\left(\omega_{1}, \omega_{2}\right)=\iint \mathbf{h}(\tau, t) \mathrm{e}^{-i\left(\omega_{1} \tau+\omega_{2} t\right)} \mathrm{d} \tau \mathrm{d} t
$$

Inversely, we can write the matrix $\mathbf{h}(\tau, t)$ from the bifrequency function $\mathbf{H}\left(\omega_{1}, \omega_{2}\right)$ as

$$
\mathbf{h}(\tau, t)=\frac{1}{4 \pi^{2}} \iint_{-\infty}^{+\infty} \mathbf{H}\left(\omega_{1}, \omega_{2}\right) \mathrm{e}^{i\left(\omega_{1} \tau+\omega_{2} t\right)} \mathrm{d} \omega_{1} \mathrm{~d} \omega_{2}
$$

By considering the dynamic responses to truncated sample function force $\mathbf{w}_{T}^{(k)}(t)$, it was been shown that dynamic response power spectral density is obtained from the input power spectral density by:

$$
\begin{gathered}
4 \pi^{2} \mathbf{S}_{\mathbf{x x}}(\omega)= \\
\int_{-\infty}^{+\infty} \mathbf{H}\left(\omega_{1}, \omega-\omega_{1}\right) \mathbf{S}_{\mathbf{w w}}(\omega) \mathbf{H}^{*}\left(\omega_{1}, \omega-\omega_{1}\right) \mathrm{d} \omega_{1}
\end{gathered}
$$

Finally, the computation of $\mathbf{S}_{\mathbf{x x}}(\omega)$ depends on the bispectrum function $\mathbf{H}\left(\omega_{1}, \omega_{2}\right)$ which could only be obtained by numerical scheme.

\section{COMPUTATIONAL PROCEDURE}

\section{General principal}

From a practical point of view, there are two ways to calculate dynamic responses of the system. The first one consists in computing the bi-spectrum matrix $\mathbf{H}\left(\omega_{1}, \omega_{2}\right)$ before determining the specific dynamic responses. The second way is a direct computation of these dynamic responses. For these two approaches, the problem is to compute the dynamic response of the system to external forces. Indeed, for the first purpose, the response $\mathbf{y}_{j}\left(\omega_{1}, t\right)$ of the system to excitation vector $\mathbf{s}_{j}\left(\omega_{1}, t\right)$ whose $j$-th component is equal to $\exp \left(i \omega_{1} t\right)$ while the others are equal to zero needs to be considered. Then both the excitation and the response vectors are given by

$$
\begin{gathered}
\mathbf{s}_{j}\left(\omega_{1}, t\right)=\boldsymbol{\sigma}_{j} e^{i \omega_{1} t} \\
\mathbf{y}_{j}\left(\omega_{1}, t\right)=\int \mathbf{h}(\tau, t) \boldsymbol{\sigma}_{j} e^{i \omega_{1}(t-\tau)} \mathrm{d} \tau
\end{gathered}
$$

where

$$
\left(\boldsymbol{\sigma}_{j}\right)_{k}=\delta_{j k}
$$

Equation (10) represents the Fourier transform of the impulse response matrix $\mathbf{h}(\tau, t)$ with respect to $\tau$, then

$$
\mathbf{y}_{j}\left(\omega_{1}, t\right)=\mathbf{H}\left(\omega_{1}, t\right) \boldsymbol{\sigma}_{j} e^{i \omega_{1} t}
$$

Now, by computing the Fourier transform of $\mathbf{y}_{j}\left(\omega_{1}, t\right)$ with respect to $t$, we obtain

$$
\begin{gathered}
\mathbf{Y}_{j}\left(\omega_{1}, \omega\right)=\int \mathbf{H}\left(\omega_{1}, t\right) \boldsymbol{\sigma}_{j} e^{i\left(\omega_{1}-\omega\right) t} \mathrm{~d} t \\
\mathbf{Y}_{j}\left(\omega_{1}, \omega\right)=\mathbf{H}\left(\omega_{1}, \omega-\omega_{1}\right) \boldsymbol{\sigma}_{j}
\end{gathered}
$$

Therefore, the columns of the matrix $\mathbf{H}\left(\omega_{1}, \omega-\omega_{1}\right)$ can be known by the computation of the response spectrum subjected to $e^{i \omega_{1} t}$ applied at each degree of freedom of the system. With the knowledge of the power spectral density $\mathbf{S}_{\mathbf{w w}}(\omega)$, one can finally compute the power spectral density of the output dynamic response $\mathbf{S}_{\mathbf{x x}}(\omega)$.

The second way, i.e. the direct computation of the response characteristics, is based on Monte Carlo simulations. Thus, we need to generate samples of input forces. This can be performed by imposing the spectral density of the input with stochastic phases uniformly distributed over $[0,2 \pi[$. This procedure leads to a Gaussian process for the input.

\section{Practical computational scheme}

In order to compute stationary responses, we use an iterative spectral method previously defined and described for determinist external excitations [7-8] and random ones [9] in the context of rotor dynamics. To this end, the matrix equation (1) is rewritten in the modal basis obtained by considering the average characteristic of the system. This modal basis is computed with the mass matrix $\mathbf{M}$ and the mean-time stiffness matrix $\mathbf{K}_{\text {avg }}$ given by

$$
\mathbf{K}_{\mathrm{avg}}=\frac{1}{T_{z}} \int_{0}^{T_{z}} \mathbf{K}(t) \mathrm{d} t
$$

It leads to the eigenfrequencies $\left\{\omega_{j}\right\}$ and the associated eigenvectors $\mathbf{v}_{j}$ normalized by the mass matrix, such as:

$$
\begin{gathered}
\mathbf{v}_{i}^{\mathrm{T}} \mathbf{M} \mathbf{v}_{j}=\delta_{i}^{j} \\
\mathbf{v}_{i}^{\mathrm{T}} \mathbf{K}_{\mathrm{avg}} \mathbf{v}_{j}=\omega_{j}{ }^{2} \delta_{i}^{j}
\end{gathered}
$$

By introducing the modal coordinates vector $\mathbf{q}$ with $\mathbf{x}=\mathbf{V q}$ with $\mathbf{V}=\left\{\mathbf{v}_{j}\right\}$, and assuming that the damping matrix is proportional to the mass or stiffness matrices, we obtain the following equation

$$
\mathbf{m} \ddot{\mathbf{q}}+\mathbf{c} \dot{\mathbf{q}}+\mathbf{k q}+\mathbf{g}(t) \mathbf{q}=\mathbf{s}(t)
$$

In this equation, $\mathbf{m}, \mathbf{c}$ and $\mathbf{k}$ are diagonal matrices with the following values $m_{j j}=1, c_{j j}=2 \zeta_{j} \omega_{j}$ and $k_{j j}=$ 
$\omega_{j}^{2}$ respectively, with $\zeta_{j}$ an equivalent modal damping coefficient for the $j$-th eigenmode. $\mathbf{g}(t)$ represents the periodical counterpart of the modal stiffness matrix defined by $\mathbf{V}^{\mathrm{T}}\left(\mathbf{K}(t)-\mathbf{K}_{\mathrm{avg}}\right) \mathbf{V}$. Notice that the matrix equation remains coupled by this parametric excitation. Finally and concerning the external forces, the only introduced ones are the stochastic processes $\mathbf{s}(t)=\mathbf{V}^{T} \mathbf{w}(t)$. Transferring the parametrical terms to the right-hand side, equation (18) becomes

$$
\mathbf{m} \ddot{\mathbf{q}}+\mathbf{c} \dot{\mathbf{q}}+\mathbf{k q}=\mathbf{s}(t)-\mathbf{g}(t) \mathbf{q}(t)
$$

Assuming that the system is asymptotically stable, i.e. it is outside of parametric instabilities, free responses vanish as time increases. Then, by retaining only stationary terms, the Fourier transform of equation (19) leads to

$$
\left(-\omega^{2} \mathbf{m}+i \omega \mathbf{c}+\mathbf{k}\right) \widehat{\mathbf{q}}(\omega)=\hat{\mathbf{s}}(\omega)-[\hat{\mathbf{g}} * \widehat{\mathbf{q}}](\omega)
$$

where $\widehat{\mathbf{q}}, \hat{\mathbf{s}}, \hat{\mathbf{g}}$ are the Fourier transform of $\mathbf{q}, \mathbf{s}, \mathbf{g}$ respectively, and $*$ denotes the convolution product. In order to solve equation (20), we retain an efficient and proven iterative procedure leading at the $(n+1)$-th iteration

$$
\widehat{\mathbf{q}}_{n+1}(\omega)=\widehat{\mathbf{q}}_{0}(\omega)-\mathbf{H} \cdot\left[\hat{\mathbf{g}} * \widehat{\mathbf{q}}_{n}\right](\omega)
$$

In this equation, $\mathbf{H}(\omega)$ is the modal frequency response complex functions matrix and $\widehat{\mathbf{q}}_{0}$ is the initial response which corresponds to the modal frequency response of the time-invariant system. They are given by

$$
\begin{gathered}
\mathbf{H}(\omega)=\left(-\omega^{2} \mathbf{m}+i \omega \mathbf{c}+\mathbf{k}\right)^{-1} \\
\widehat{\mathbf{q}}_{0}(\omega)=\mathbf{H}(\omega) \hat{\mathbf{s}}(\omega)
\end{gathered}
$$

This iterative procedure is applied until the error of the root mean squared values between two successive iterations is lower than a prescribed sufficiently small value. Up to now, and in various context, no convergence problem has been found.

Finally, the frequency response $\mathbf{Y}_{j}\left(\omega_{1}, \omega\right)$, equation (14), can be numerically obtained. In a same way, the spectral characteristics of the dynamic response can be also directly done by Monte Carlo simulations.

\section{ILLUSTRATIVE EXAMPLES}

\section{The single-degree-of-freedom gear modelling}

As an application, a standard single-degree-offreedom spur parallel gear pair running at constant speed is introduced. The geared system is excited by both parametric excitation and a random external excitation. The parametric excitation is defined as a periodically timevarying stiffness. Thus, the equation of motion is written as

$$
m \ddot{x}+c \dot{x}+\overline{k(t)} x+g(t) x(t)=w^{(k)}(t)
$$

where $x$ is the dynamic transmission error computed along the line of action, $m$ is an equivalent mass including inertia of wheels, $k$ is the time-averaged meshing stiffness acting along the line of action of the gear pair, $g(t)$ is the fluctuating part of the meshing stiffness $(k(t)-\overline{k(t)}), c$ is a viscous coefficient and $w^{(k)}$ is a sample of the excitation process. Without loss of generality, one can rewrite this equation in the following form

$$
\ddot{x}+2 \zeta \Omega \dot{x}+\Omega^{2}[1+\alpha p(t)] x(t)=s^{(k)}(t)
$$

In this equation, $\Omega$ denotes the modal frequency of the gear pair (extracted for the time-invariant system), $\zeta$ is the equivalent viscous damping ratio, $p(t)$ is a periodical function at the meshing frequency $\omega_{z}, \alpha$ governs the amplitude of the parametric excitation, and $s^{(k)}(t)$ is the random excitation divided by the equivalent mass. It consists on a zero-mean Gaussian white noise truncated over a frequency range ]0 $4 \Omega$ ] whose magnitude is $S_{0}$. Finally, the Monte Carlo simulation is performed after a frequency discretization by introducing the following pseudo random process

$$
s^{(k)}(t)=\sum_{j=1}^{j=N} A_{j} \cos \left(j \omega_{0} t-\varphi_{j}^{(k)}\right)
$$

with $A_{j}=2 \sqrt{\Omega S_{0}} / N$ and $\varphi_{j}{ }^{(k)}$ a random variable uniformly distributed over the interval [ $\begin{array}{ll}0 & 2 \pi[\end{array}$.

\section{The power spectral density response}

A typical power spectral density (PSD) of a sample of the dynamic displacement response $\mathrm{S}_{\mathrm{xx}}(\varpi)$ with $\varpi=\omega / \Omega$, is shown in figure 1 . The conditions for this response are $p(t)=\cos \left(\omega_{z} t\right)$ with $\omega_{z}=1.3 \Omega, \alpha=0.2$, and $\zeta=2 \%$. The ensemble average of the PSD with 500 samples is also shown in figure 2 for the same conditions.

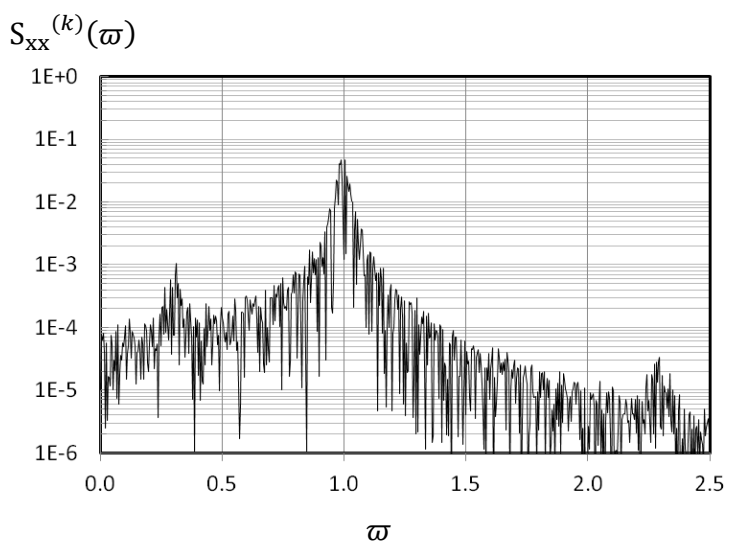

Fig.1 Typical single sample of the PSD response

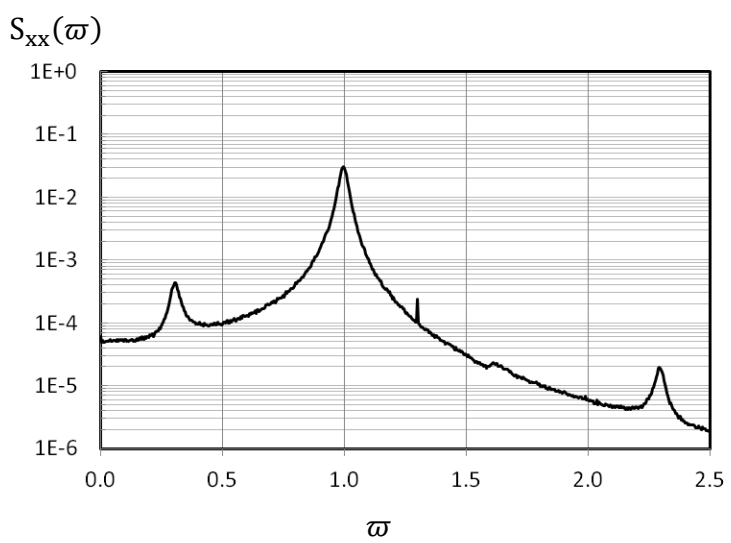

Fig.2 Ensemble average of the PSD response 
As the convolution product in equation (21) is directly performed without Fourier transform in the time domain, the calculation time was found to be very short (less then 1s CPU time on a usual laptop) which demonstrate the efficiency of the proposed method. As we can see, the filtered response of the broadband random process is clearly shown with the occurrence of three spectral peaks. The principal ones at $\varpi=1.0$ corresponds to the direct excitation of the geared system at its modal frequency by the broadband excitation. The secondary peaks at $\varpi=0.3$ and $\varpi=2.3$ correspond to the parametric effect. More generally, the supplementary peaks can be predicted localized at

$$
\omega=\left|\omega_{z} \pm \Omega\right|
$$

\section{Effect of the viscous damping ratio}

For the same conditions than the above results, but with two different damping ratio $(\zeta=1 \%$ or $2 \%)$, we compare the ensemble-averaged PSD of the response in figure 3 . As expected, it only affects all the spectral peaks in level in the same way of the dynamic response of a time constant dynamic system.

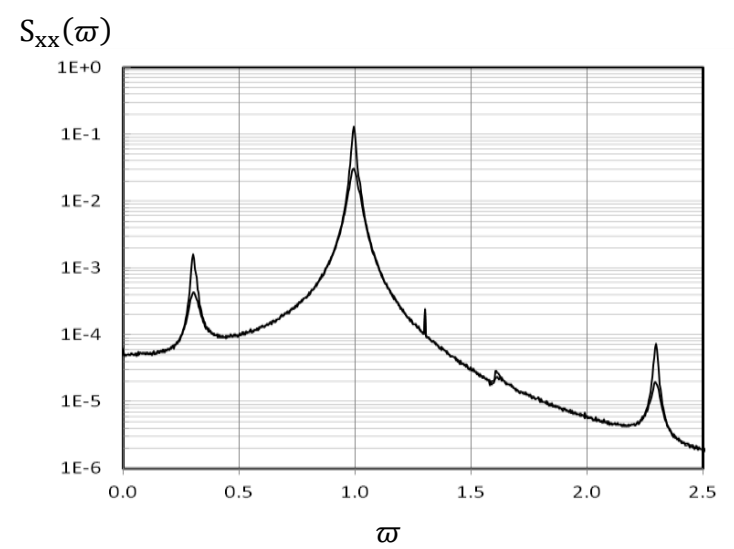

Fig.3 Ensemble average of the PSD response for $\zeta=1 \%$ or $2 \%$.

\section{Effect of the spectral content of the meshing stiffness}

In this new case the meshing stiffness is introduced as a periodic stiffness not purely harmonic. More precisely, $p(t)=\cos \left(\omega_{z} t\right)+0.6 \cos \left(2 \omega_{z} t\right)$, and figure 4 shows the result.

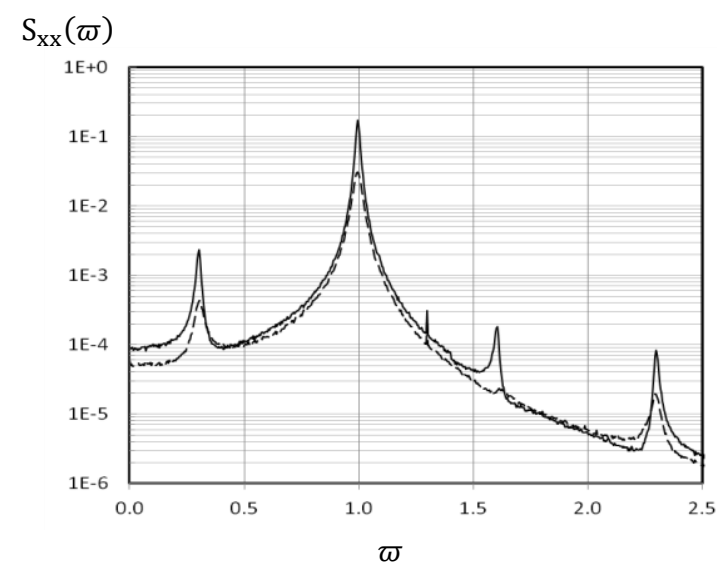

Fig.4 Ensemble average of the PSD response for $p(t)=\cos \left(\omega_{z} t\right)+0.6 \cos \left(2 \omega_{z} t\right)$ in thick line and for $p(t)=\cos \left(\omega_{z} t\right)$ in dashed line.
We can observe two differences. The first one is the clearly occurrence of a new spectral peak. It has been found centered around $\omega=\left|2 \omega_{z} \pm \Omega\right|$, i.e. for the example $\varpi=$ 1.6. The second difference consists on higher levels clearly observed on all the spectral peaks. This result demonstrates the interaction between the spectral components, which is a typical parametric effect.

\section{Effect of a low parametric excitation frequency}

Now, we consider a meshing frequency for the parametric meshing stiffness lower than the modal excitation, i.e. $\omega_{z}=0.3 \Omega$. Figures 5 and 6 show the results for two parametric excitations, $\quad p(t)=\cos \left(\omega_{z} t\right)+$ $0.6 \cos \left(2 \omega_{z} t\right)$ and $p(t)=\cos \left(\omega_{z} t\right)+0.6 \cos \left(3 \omega_{z} t\right)$.

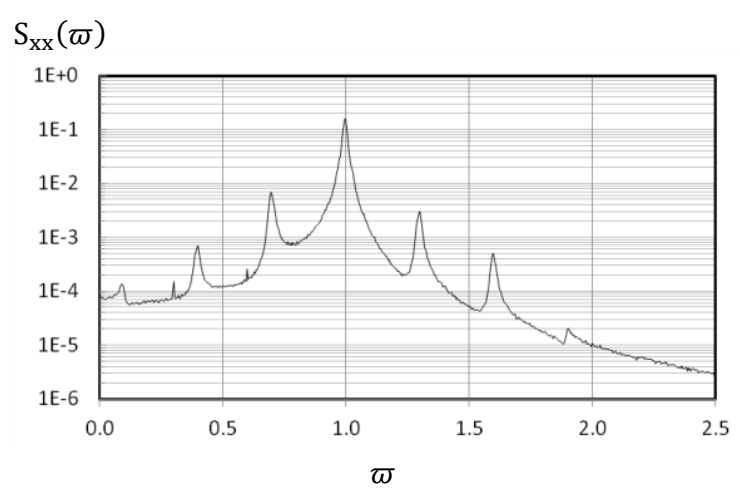

Fig.5 Ensemble average of the PSD response for $p(t)=\cos \left(\omega_{z} t\right)+0.6 \cos \left(2 \omega_{z} t\right)$

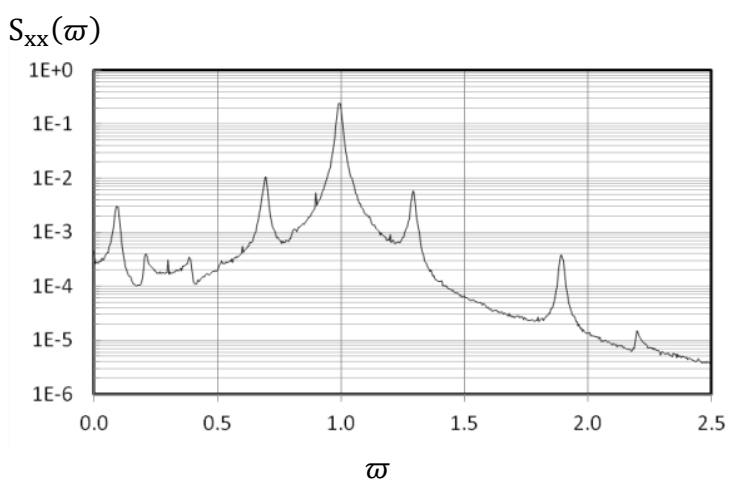

Fig.5 Ensemble average of the PSD response for $p(t)=\cos \left(\omega_{z} t\right)+0.6 \cos \left(3 \omega_{z} t\right)$

Once again, we can clearly distinguish the appearance of secondary peaks around the primary peak at $\varpi=1$. For all the cases, these spectral peaks are localized at

$$
\omega=\left|n \omega_{z} \pm \Omega\right|
$$

In this relation, $\mathrm{n}$ is a natural number. As we can observe in these figures, the dominant spectral peaks are related to the spectral content of the time-varying mesh stiffness parametric excitation. Thus, for the first simulation, the main amplifications occur for $n=1$ or 2 that is at $\varpi=1.3\left(=\varpi_{z}+1\right), 0.7\left(=\varpi_{z}-1\right), 1.6$ (= $\left.2 \varpi_{z}+1\right)$ and $0.4\left(=2 \varpi_{z}-1\right)$ and with a very low level at $0.1\left(=3 \varpi_{z}+1\right)$ and $1.9\left(=3 \varpi_{z}+1\right)$. The same result for the second case ( $n=1$ or 3 for the dominant peaks) that is localized at $\varpi=1.3\left(=\varpi_{z}+1\right), 0.7\left(=\varpi_{z}-1\right)$, $1.9\left(=3 \varpi_{z}+1\right)$, and $0.1\left(=3 \varpi_{z}-1\right)$ and with a lower level at $\varpi=0.2,0.4$ and 2.2 . 
To conclude on these phenomena, the effect of the parametric excitation on the broadband external excitation can be easily understood by considering the iterative spectral method, equation (21), which clearly displays the convolution product effect on the spectral response.

\section{CONCLUSION}

This paper focused on a spectral approach to compute the stationary response of a geared transmission system modelling both excited by the periodical meshing stiffness internal parametric excitation and by broadband white noise external excitations. In this paper, the response PSD is formally expressed as a function of the input PSD via the bispectrum functions, which are the bilinear Fourier transform of the bitemporal impulse responses. The bispectrum or more directly the PSD response are computed using an iterative spectral method developed previously for parametrical systems under deterministic external excitation and for gear applications, the whining noise prediction. In the context of rotor dynamics, the case of external random excitation has been also considered. The efficiency of the proposed method in terms of accuracy and computation time required has been demonstrated. Concerning the dynamic behavior of the geared system, the multifrequency nature of responses has been exhibited. The main result is the occurrence of spectral peaks localized at the natural frequency and its sideband governed by the harmonics of the meshing frequency according to the equation (25). Finally, peak levels are mainly governed by damping ratio and the spectral content of the meshing stiffness.

\section{ACKNOWLEDGMENT}

This work has been performed within the LabCom LADAGE (Laboratoire de dynamique des engrenages) funded by the French National Research Agency / ANR under the reference number ANR-14-LAB6-0003. The authors are also members of the LabEx CeLyA (Centre Lyonnais d'Acoustique) funded by the French Ministry of Research

\section{REFERENCES}

1. Harris, S. L. (1958). Dynamic loads on the teeth of spur gears. Proceedings of the Institution of Mechanical Engineers, 172(1), 87-112.

2. Welbourn, D. B. (1979). Fundamental knowledge of gear noise: a survey (No. IMechE-C117/79).

3. Özgüven, H. N., \& Houser, D. R. (1988). Mathematical models used in gear dynamics - a review. Journal of sound and vibration, 121(3), 383-411.

4. Perret-Liaudet, J., Carbonelli, A., Rigaud, E., Nelain, B., Bouvet, P., \& Vialonga, C. J. (2014). Modeling of gearbox whining noise (No. 2014-01-2090). SAE Technical Paper.

5. Carbonelli, A., Perret-Liaudet, J., \& Rigaud, E. (2014, September). Hammering noise modelling - Nonlinear dynamic of a multi-stage gear train. In International Gear Conference, Lyon (pp. 447-456).

6. Kadmiri, Y., Rigaud, E., Perret-Liaudet, J., \& Vary, L. (2012). Experimental and numerical analysis of automotive gearbox rattle noise. Journal of Sound and Vibration, 331(13), 3144-3157.

7. Perret-Liaudet, J. (1996). An original method for computing the response of a parametrically excited forced system. Journal of Sound and Vibration, 196(2), 165-177.

8. Carbonelli, A., Rigaud, E., \& Perret-Liaudet, J. (2016). Vibro-Acoustic Analysis of Geared SystemsPredicting and Controlling the Whining Noise. In Automotive NVH Technology (pp. 63-79). Springer International Publishing.

9. Bachelet, L., Driot, N., \& Perret-Liaudet, J. (2008). A spectral method for describing the response of a parametrically excited system under external random excitation. Journal of Computational and Nonlinear Dynamics, 3(1), 011008. 